\title{
Instabilité génétique chez des enfants traités par chimiothérapie pour une leucémie
}

L'idée que les cancers humains résultent d'une accumulation de mutations géniques somatiques acquises dans une lignée cellulaire clonale semble maintenant acquise. Les gènes en cause codent souvent pour des protéines qui jouent un rôle important dans les processus de prolifération cellulaire, de différenciation, d'apoptose et/ou de réparation de l'ADN. Le modèle de développement du cancer colo-rectal en plusieurs étapes d'acquisitions de modifications géniques en est une excellente illustration [1].

En revanche, les mécanismes à l'origine de l'apparition de ces mutations somatiques multiples sont très débattus depuis des décennies. Le taux de mutations spontanées dans une cellule somatique normale n'est que de l'ordre de $10^{-5}$ à $10^{-7}$ par division cellulaire in vivo, et ne peut donc expliquer le nombre élevé de mutations détectées dans certains cancers humains. La question se pose donc de savoir comment l'accumulation d'altérations géniques coopératives peut survenir au sein d'une même cellule dans un délai raisonnable à l'échelle d'une vie humaine. Deux mécanismes sont proposés :

1) Une augmentation préalable du taux de mutations intrinsèques, autrement dit une instabilité génétique, dans des cellules non malignes (ou prémalignes) faciliterait l'émergence du processus malin dans une cellule ou un petit nombre d'entre elles [2].

2) Certaines mutations spontanées (survenant avec une fréquence normale) sélectionnent un clone cellulaire en lui conférant un avantage prolifératif considérable. L'augmentation du nombre de divisions cellu- laires de ce clone sélectionné permettrait l'apparition, même en l'absence d'instabilité génétique, d'un nombre élevé de mutations [3]. Dans les cellules tumorales, ces deux processus sont souvent intriqués et, lorsque de multiples anomalies géniques sont détectées, la primauté de l'instabilité génétique sur l'avantage sélectif dans la transformation maligne ne peut être déterminée.

En revanche, dans les cellules humaines non malignes, l'existence d'une instabilité génétique peut être appréciée indirectement par l'étude de gènes marqueurs dont l'altération entraîne un phénotype facilement sélectionnable. Ainsi, l'augmentation de la fréquence d'un phénotype dit " mutateur» a été mise en évidence chez des enfants traités par une polychimiothérapie intensive pour leucémie aiguë lymphoblastique (LAL) [4, 5]. Ces résultats suggéraient, mais sans le démontrer formellement, l'existence d'une instabilité génétique provoquée par la chimiothérapie et donc susceptible d'augmenter le risque de néoplasie secondaire chez ces enfants. Une étude récente [6] démontre clairement que les cellules T non malignes d'enfants traités pour une LAL de la lignée B présentent une instabilité génétique caractérisée par des mutations multiples d'un gène marqueur au sein d'un même clone cellulaire $\mathrm{T}$.

Le gène domestique codant pour l'hypoxanthine-guanine phosphoribosyltransférase (HPRT) est couramment utilisé pour les études in vivo de mutagenèse en raison de la facilité de sélection des mutants. L'enzyme HPRT intervient dans la voie de recyclage des nucléotides puriques à partir des bases puriques hypoxanthine et guanine. Elle phosphoribosyle également les analogues des purines, tels que le 6-thioguanine, ce qui induit un effet cytotoxique. Ainsi, en présence de 6-thioguanine, seules les cellules ayant une mutation inactivante du gène HPRT sont capables de proliférer [7]. De plus, chez les enfants atteints de LAL, les analogues des purines, tels que le 6-thioguanine (Lanvis ${ }^{\mathrm{R}}$ ) et le 6-mercaptopurine (Purinéthol ${ }^{\mathrm{R}}$ ), sont fréquemment utilisés dans les traitements d'entretien et sont donc susceptibles de favoriser la sélection des cellules dont l'activité HPRT est déficiente.

Finette, et al. ont déterminé la fréquence des clones lymphocytaires $\mathrm{T}$ ayant un phénotype HPRT-muté chez 103 enfants ayant une LAL de la lignée B (19 au moment du diagnostic initial avant tout traitement par chimiothérapie, 32 en rémission complète, et 58 lors de la rechute avant traitement) et 49 enfants sains appariés selon l'âge [6]. Quand la fréquence de mutation du gène HPRT était très élevée, la caractérisation au niveau nucléique de ces mutations a été effectuée [8] et couplée au séquençage de la région hypervariable CDR3 de la chaîne $\beta$ du récepteur des lymphocytes $\mathrm{T}$ TCR $\beta$, permettant ainsi d'attribuer ces mutations du gène HPRT à des clones $\mathrm{T}$ spécifiques [9]. Les résultats confirment que la fréquence des cellules $\mathrm{T}$ ayant un phénotype HPRTmuté est nettement plus élevée chez les patients traités par chimiothérapie (LAL en rémission ou en rechute) que chez ceux étudiés avant traitement ou chez les sujets témoins (entre ces deux derniers groupes, les fréquences de mutation ne sont pas significativement différentes).

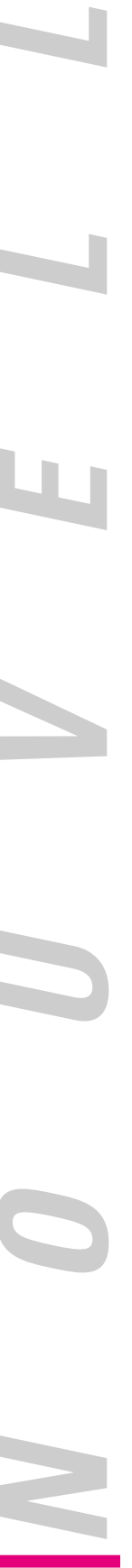


Chez 15 des 24 patients ayant des fréquences de mutations très élevées, 182 colonies de cellules $\mathrm{T}$ mutantes ont été isolées indépendamment afin d'effectuer le séquençage du gène HPRT et de la région CDR3 du gène TCR $\beta$. On observe pour 4 patients ( 1 cas de rémission et 3 de rechutes) des mutations multiples sur le même allèle du gène marqueur HPRT: délétions de taille variable, substitutions nucléotidiques, plus rarement insertions. De plus, ces mutations sont détectées dans des clones $\mathrm{T}$ différents, 1 à 9 clones $\mathrm{T}$ distincts comportent chacun 1 à 3 mutations différentes du gène HPRT.

On peut calculer que pour un taux de mutation spontanée de $10^{-5}$ à $10^{-7}$ par génération cellulaire, il faudrait environ $10^{10}$ à $10^{21}$ générations cellulaires pour accumuler 2 ou 3 mutations sur le même allèle d'un gène. Il est clair que de tels événements mutationnels ne peuvent survenir dans un délai raisonnable à l'échelle d'une vie humaine. Même dans l'hypothèse où une expansion clonale massive après la chimiothérapie ait favorisé la survenue de mutations multiples dans un clone cellulaire $\mathrm{T}$, ceci ne pourrait pas expliquer la présence de plusieurs mutations au sein du même allèle d'un seul gène.

Il apparaît donc que les mutations observées dans cette étude reflètent in vivo l'évolution d'une instabilité génétique restreinte à certains clones cellulaires. Cette perte de l'intégrité génomique apparaît dans des cellules $\mathrm{T}$ non malignes, au décours de poly- chimiothérapies intensives qui représentent une exposition séquentielle à des effets cytotoxiques et mutagènes. Les données issues des divers modèles expérimentaux plaident en faveur d'un lien de causalité dans cette association entre chimiothérapies utilisées dans certaines leucémies et instabilité génétique.

Il reste à prouver le rôle déterminant dans l'induction de néoplasies secondaires ou dans la résistance aux traitements de cette instabilité génétique induite par la chimiothérapie dans des cellules non malignes. Quoi qu'il en soit, l'identification de clones cellulaires ayant un fort potentiel de mutations fournit un matériel d'étude idéal pour la recherche de gènes impliqués dans le contrôle de l'intégrité du génome humain. Les données expérimentales in vitro, ou in vivo dans les modèles murins, et certains syndromes héréditaires comme l'ataxie-télangiectasie [10], le xeroderma pigmentosum $(\mathrm{m} / \mathrm{s} 1998$, $\left.n^{\circ} 11, p .1289\right)$ ou le cancer colique héréditaire non polyposique permettent déjà de pressentir certains gènes candidats comme p53 [11], ATM, BRCA1, BRCA2 ou les gènes MMR...

1. Kinzler KW, Vogelstein B. Lessons from hereditary colorectal cancer. Cell $1996 ; 87$ : 159-70.

2. Lengauer C, Kinzler KW, Vogelstein B. Genetic instabilities in human cancers. Nature 1998 ; 396 : 643-9.

3. Tomlinson I, Bodmer W. Selection, the mutation rate and cancer: ensuring that the tail does not wag the dog. Nat Med 1999 ; 5 : 11-2.
4. Hirota H, Kubota M, Adachi S, et al. Somatic mutations at T-cell antigen receptor and glycophorin A loci in pediatric leukemia patients following chemotherapy: comparison with HPRT locus mutation. Mutat Res 1994 ; 315 : 95-103.

5. Koishi S, Kubota M, Sawada M, et al. Biomarkers in long survivors of pediatric acute lymphoblastic leukemia patients: late effects of cancer chemotherapy. Mutat Res 1998 ; 422 : 213-22.

6. Finette BA, Homans AC, Albertini RJ. Emergence of genetic instability in children treated for leukemia. Science 2000 ; 288 : 514-7.

7. Finette BA, Sullivan LM, O’Neill JP, Nicklas JA, Vacek PM, Albertini RJ. Determination of hprt mutant frequencies in T-lymphocytes from a healthy pediatric population: statistical comparison between newborn, children and adult mutant frequencies, cloning efficiency and age. Mutat Res $1994 ; 308: 223-31$

8. Finette BA, O'Neill JP, Vacek PM, Albertini RJ. Gene mutations with characteristic deletions in cord blood $\mathrm{T}$ lymphocytes associated with passive maternal exposure to tobacco smoke. Nat Med $1998 ; 4$ : 1144-51.

9. Albertini RJ, Nicklas JA, Skopek TR, Recio L, O'Neill JP. Genetic instability in human T-lymphocytes. Mutat Res 1998 ; 400 : 381-9.

10. Bay JO, Uhrhammer N, Hall J, Stoppa- Lyonnet D, Bignon YJ. Fonctions de la protéine ATM et aspects phénotypiques de l'ataxie télangiecatsie. Med Sci 1999 ; 15 : 1086-95.

11. Soussi T. Cycle cellulaire et apoptose : le gène suppresseur de tumeur p53. Med Sci 2000 ; 16 : 469-72.

\section{Hélène Poirel}

Service d'hématologie biologique. Hôpital Avicenne, 125, rue de Stalingrad, 93009 Bobigny Cedex, France.

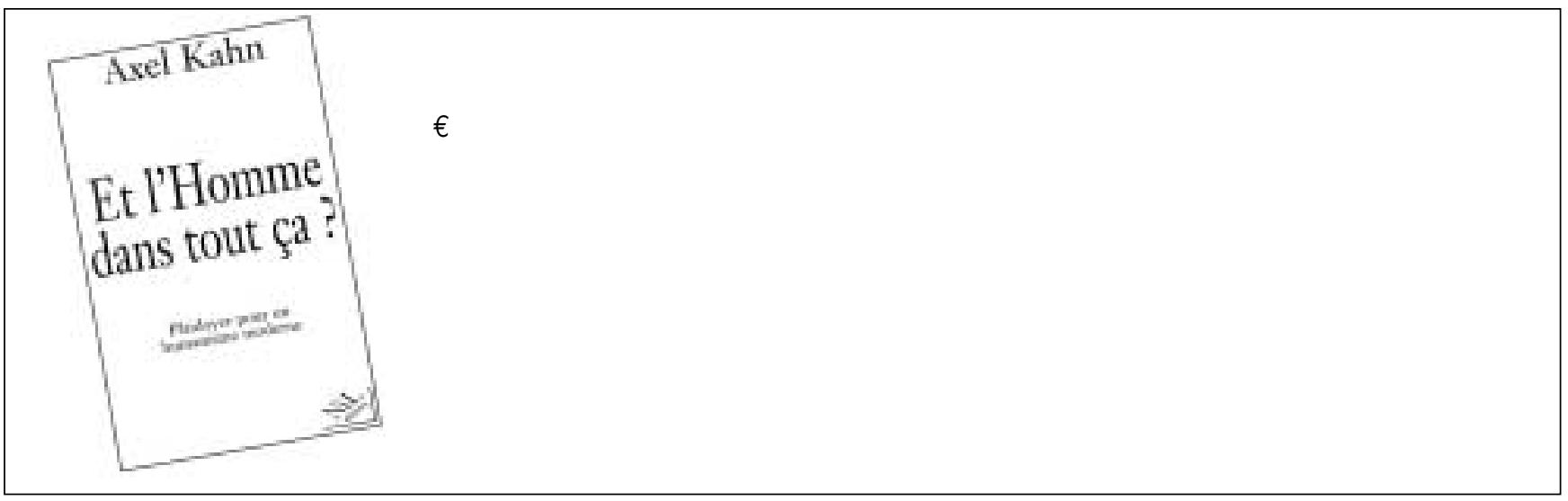

